Draft VERSION OCTOBER 17, 2017

Typeset using LATEX preprint2 style in AASTeX61

\title{
INFORMATION RETRIEVAL AND RECOMMENDATION SYSTEM FOR ASTRONOMICAL OBSERVATORIES
}

\author{
Nikhil Mukund,${ }^{1}$ Saurabh Thakur, ${ }^{2}$ Sheelu Abraham, ${ }^{1}$ A. K. Aniyan, ${ }^{3,}{ }^{4}$ Sanjit Mitra, ${ }^{1}$ \\ Ninan Sajeeth Philip, ${ }^{5}$ Kaustubh Vaghmare, ${ }^{1}$ And D. P. Acharjya ${ }^{2}$
}

\author{
${ }^{1}$ Inter-University Centre for Astronomy and Astrophysics (IUCAA), Post Bag 4, Ganeshkhind, Pune 411 007, India \\ ${ }^{2}$ Vellore Institute of Technology, Tamil Nadu, India \\ ${ }^{3}$ Department of Physics and Electronics, Rhodes University, Grahamstown, South Africa \\ ${ }^{4}$ SKA South Africa, $3^{\text {rd }}$ Floor, The Park, Cape Town, South Africa \\ ${ }^{5}$ Department of Physics, St. Thomas College, Kozhencherry, Kerala, India
}

\begin{abstract}
We present a machine learning based information retrieval system for astronomical observatories that tries to address user defined queries related to an instrument. In the modern instrumentation scenario where heterogeneous systems and talents are simultaneously at work, the ability to supply with the right information helps speeding up the detector maintenance operations. Enhancing the detector uptime leads to increased coincidence observation and improves the likelihood for the detection of astrophysical signals. Besides, such efforts will efficiently disseminate technical knowledge to a wider audience and will help the ongoing efforts to build upcoming detectors like the LIGO-India etc even at the design phase to foresee possible challenges. The proposed method analyses existing documented efforts at the site to intelligently group together related information to a query and to present it on-line to the user. The user in response can further go into interesting links and find already developed solutions or probable ways to address the present situation optimally. A web application that incorporates the above idea has been implemented and tested for LIGO Livingston, LIGO Hanford and Virgo observatories.
\end{abstract}

Keywords: Astronomical instrumentation, methods and techniques, methods: data analysis 


\section{INTRODUCTION}

Data mining in the big data framework often encounters difficulty in both extracting the relevant information from the data and in coming up with meaningful interpretations in a highly reliable fashion (Fan et al. 2014; Wu et al. 2014; Khan et al. 2014). In many situations, the data comes in a format which is not suitable to store in relational databases of coherent hierarchy (Stephens et al. 2015). The methods in which the data is stored and associated with different entities also pose the challenge in mining required information from it. For example, in a gravitational wave observatory, there will be a core science dataset with plenty of meta-data on the observation and a variety of other auxiliary datasets collected from various sensors and actuators.

Even though data mining methods like association analysis, clustering and other machine learning techniques exist, the presentation of unstructured data into these algorithms and inference generation is not a trivial task (Han et al. 2011). Generation of insights from big data with recommendation systems which are based on learning from unstructured text data (Pazzani \& Billsus 2007) tackle these challenges at large (LaValle et al. 2011; Hu et al. 2014). Descriptive recommendations and information retrieval (Sigurbjörnsson \& Van Zwol 2008; Gretzel et al. 2004) have recently have recently gained popularity and have been applied to areas like travel recommendation systems (Gretzel et al. 2004) and content personalization systems (Liang et al. 2006). Besides commercial applications, text summarization based content recommendation (Hassan et al. 2009) is an interesting area which has high level of applicability in different areas of science and research (Miner et al. 2012; Kerzendorf 2017). Unlike conventional rank based search systems, these do not perform topical modeling and rank topics of recurring interest (Zoghbi et al. 2013).
Topical modeling is usually done for retrieving information from a single website with multiple topics. The challenge is when different topics in a single site may be weakly linked to each other (Cointet \& Roth 2010). While there could be already known relations among different entities, the process of data mining and better data representation can reveal the latent unforeseen linkages among different topical entities (Behrens \& Bassu 2006).

Large science projects, especially astronomical observatories, have plenty of data about telescope operations, scheduling, maintenance and general observational activities all logged in text form. Over the years, these logbook entries will accumulate almost all the aspects of the instruments in the observatory. Although the key technologies are changing rapidly, the fundamental principles involved in construction and maintenance at observatories are getting altered at a less rapid rate. This fact necessitates the need for keeping a record of activities carried out over the years for prompt diagnostics. Projects like SKA, TMT, LIGO, SALT, JWST also require extensive internal coordination. These typically are a collaboration consisting of thousands of scientists whose research can span area like instrument fabrication, installation, commissioning, characterization, maintenance, upgrade, data analysis and parameter estimation. Often their time span spread across few decades and thus generate information whose volume and complexity cannot be handled effectively by traditional search engine backed information processing tools. On the positive side, analysis of such big data volumes can yield powerful insights into the inherent trends and fluctuations within the concerned project.

In this paper, we demonstrate natural language processing(NLP) backed knowledge rediscovery (Ricci et al. 2011) by making use of the open source logbook data from the Laser Inter- 
ferometric Gravitational Observatory (LIGO). This is a novel approach to observational astronomy, and the developed software is made available for the public through a web application named Hey $\mathbf{L I G O}^{1}$. We also show the application of descriptive content based recommendations to compare common issues among multiple observatories. These methods are generally scalable and will be very useful in the event of upcoming projects like the Square Kilometer Array (SKA) and upcoming LIGO-India detector.

We have organized the paper as follows: in section two we describe the methodology adopted to convert raw data to useful and representable information. Section three provides the details of data used in our analysis. Features of the recommendation system are outlined in section four. In the last part, we apply the scheme to various gravitational wave observatories around the world and discuss the results obtained.

\section{CONTEXTUAL LEARNING OF UNSTRUCTURED DATA}

Structured data is highly organized and usually resides in a relational database schema. But unstructured data refer to information that does not follow the traditional database scheme. For example, e-mails, web pages, business documents, FAQ's, etc. are some examples of unstructured data. They include text and even multimedia content. So processing of such information is an energy and time consuming task.

This section briefly describes the manner in which the unstructured textual data is acquired, processed and finally given structure. Moreover, it also enumerates the various steps involved in the development of a machine learning model which is used to differentiate between the available textual data points contextually. Finally,

\footnotetext{
1 heyligo.gw.iucaa.in
}

the model is used to perform clustering over all the textual data, thus, adding a structure for ease of access. Figure 1 shows the schematic representation of our web interface that is used to implement the scheme.

The unstructured dataset that we use is in the form of textual web pages. These pages have an identical HTML structure and defined attributes for every data point. Due to this same structure and open source nature of the web pages, it is possible to write a script which can extract each attribute from the HTML source code and organize the complete information into a data frame. A data frame is a tabular structure with columns as attributes and rows as individual data points. The first part of our algorithm does data acquisition using the python package "Beautiful Soup" to retrieve information from web pages by searching through all the new posts and related data. It saves it into relevant files for later utilization when the need arises.

Once the data is stored locally, non-essential attributes are removed, textual time stamps are converted to system timestamps, duplicate data points are removed/combined, and the resulting data is passed on to the text processing unit. A vocabulary for our data is generated by converting the unstructured data into stem words. For that, we have removed all special characters and punctuations such as !, @, \#, \$, \%,*,\&, , , , (, ) etc. All non-English words and other HTML tags, URLs are also excluded from the data. The text is then tokenized (Huang et al. 2007) by splitting the strings of text into a list of words called tokens. To reduce the redundancy in the vocabulary, it is important to convert the related token forms and their derivatives to a common base stem by a process known as stemming (Smirnov 2008).

In the process of embedding, the textual data is converted into vectors which can be easily handled by the computer (Li et al. 2015). 


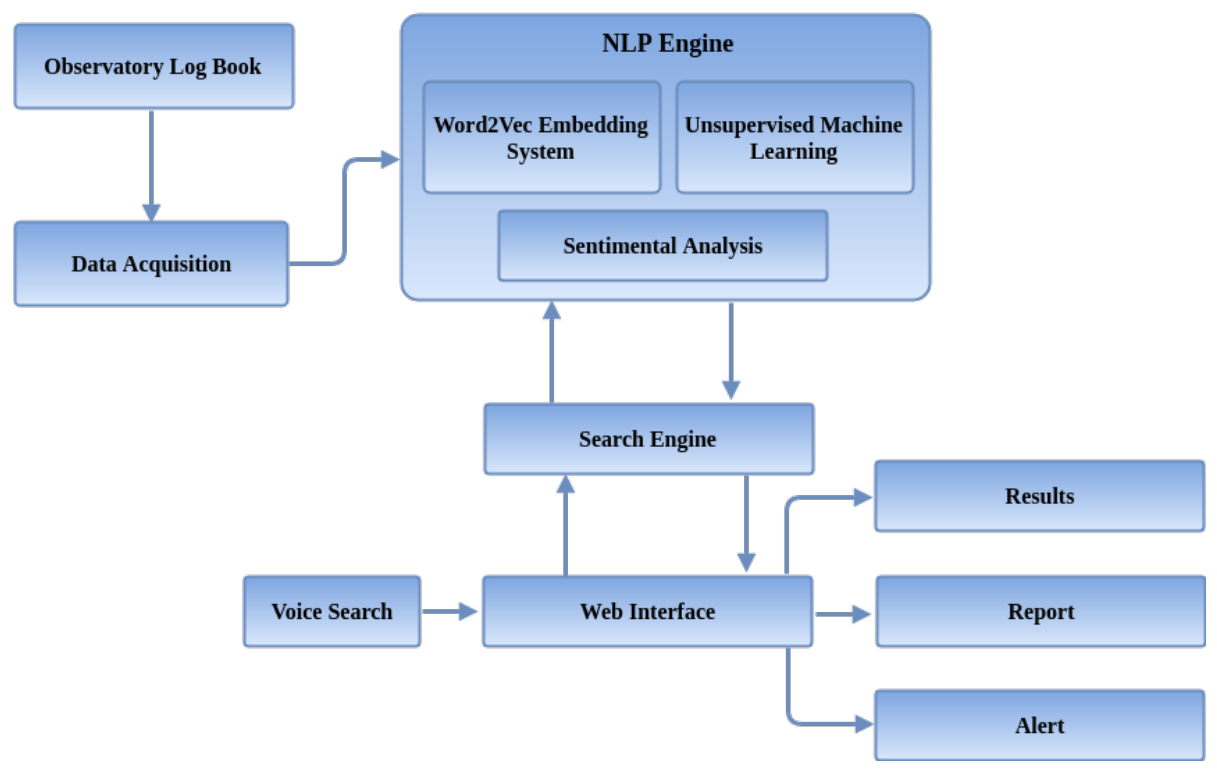

Figure 1. Data Flow through various components in the system

There exist various embedding algorithms like One-Hot Encoding (Harris \& Harris 2012) and Term Frequency Inverse Document Frequency (TFIDF) Leskovec et al. (2014) etc for this process. Not all methods can capture the contextual differences between the words. However, a recent breakthrough in the field of Natural Language Processing incorporates neural networks that can learn the vector values for each word by iterating over the text multiple times using a gradient based algorithm (Mikolov et al. 2013a,b). Bengio et al. (2003) have coined the term word embeddings with a neural language model to train them with the model's parameter.

One of the commonly used tools to convert words into vectors is word2vec described in Mikolov et al. (2013a). Word2vec has a single hidden layer, fully connected neural network that takes a large text corpus as input and produces a higher dimensional vector for each unique word in the corpus. Words which share common contexts in the corpus are located close to each other in the vector space. Word2vec models do not consider word order and can capture semantic information between words in a very efficient way (Ling et al. 2015). With the help of Word2vec embeddings, a computer can differentiate between words of different types. Word2vec implements two computationally less expensive models known as Continuous Bag of Words (CBOW) and a Skip Gram model (Mikolov et al. 2013a) to learn word embeddings. The representation of a corpus of text or an entire document in the form of a list of words (Multiset) is referred to as Bag of Words representation (Markov \& Larose 2007). The algorithm essentially tries to predict the target based on a set of context words (Mikolov et al. 2013a,b).

The model that we have used in this work is the Skip Gram model. The basic architecture of the skip gram model is shown in Figure 2. This representation is similar to CBOW model, but instead of predicting the target word, it predicts the context words based on a given target word (Mikolov et al. 2013b). Thus, the model maximizes the probability for classification of a word based on another word in the same sentence (Mikolov et al. 2013a). Thus the vector representation is capable of capturing the semantic meanings of the words from a sequence 


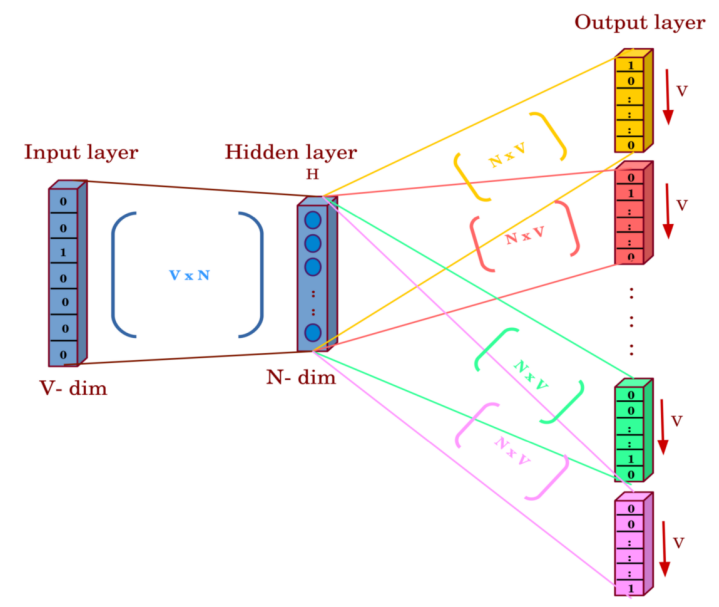

Figure 2. Skip-Gram Model

of training words $w 1, w 2, \ldots, w T$ and their contexts $c$. The algorithm can be briefed as follows. First the words are applied to as an input to a log-linear classifier where the objective is to maximize the average log probability given by,

$$
\mathcal{L}=\frac{1}{T} \sum_{t=1}^{T} \sum_{-c \leq j \leq c, j \neq 0} \log P\left(w_{t+j} \mid w t\right)
$$

Larger value of $c$ can result in higher accuracy but requires more training time (Mikolov et al. 2013b). To obtain the output probability, $P\left(w_{o} \mid w_{i}\right)$, the model estimates a matrix which maps the embeddings into $V$-dimensional vector $O_{w_{i}}$. Thus the probability of predicting the word $w_{o}$ given the word $w_{i}$ is defined using the softmax function:

$$
P\left(w_{o} \mid w_{i}\right)=\frac{\exp \left(O_{w_{i}}\left(w_{o}\right)\right)}{\sum_{w \epsilon V} \exp \left(O_{w_{i}}(w)\right)}
$$

where $V$ is the number of words in the vocabulary (Ling et al. 2015; Mikolov et al. 2013b). But this formulation is computationally intensive for larger vocabularies. This is solved in word2vec by using the hierarchical softmax function (Morin \& Bengio 2005) or with negative sampling approach (see this for more details; Goldberg \& Levy 2014).
After embedding all words, every data point is represented as the average of all the word vectors of the words present in it. A Nearest Neighbor Algorithm (Andoni \& Indyk 2006) is then used to cluster these data point vectors to respective clusters efficiently. The optimal number of clusters is estimated iteratively until it is observed that the accuracy peaked, which in our case was found to be $1 / 5^{\text {th }}$ of the vocabulary of our model. We used the Python implementation of Scikit - learn package for doing the nearest neighbor algorithm.

Even after NLP classification, we observed that quite a number of relevant posts were left out unobserved and so we added one more layer of processing by analyzing the overall emotional content of the reports. We used the AFINN lexicon (Nielsen 2011) consisting of a collection of 2477 words each with an associated integer value ranging between -5 to +5 representing transition from negative to positive sentiment. Modifying the word valence and appending the lexicon with technical words that better represent the associated sentiments was found to provide better results. For example, LIGO specific application will associate terms like 'lockloss' and 'scatter noise' with negative sentiment while 'new filter installed' would mean something positive.

The prototype is designed so that the users can query for information through a web interface. The stem words in the query are identified and the vector is projected into the previously modeled word vector space. The nearest neighbor model retrieves the top neighbors for the query vector, and are then displayed as the search results on the web interface. The search result is then filtered to check for the presence of the query words in either the title or in its content to weed out false positives. In Figure 3, we have shown a simple search query displayed on the web interface. The different features in- 
corporated in the web page is described in Section 4.

\section{GRAVITATIONAL WAVE OBSERVATORIES}

GW interferometers (IFOs) have been in operation for the past few decades and have made the first direct detection of merging binary black holes (Abbott et al. 2016b). The complex nature of this multi-physics experiment requires scientists from multiple domain of expertise to work together and share information. Rigorous commissioning and characterization efforts have been carried over a span of two decades to reach the current level of sensitivity. LIGO, Virgo, GEO600 and KAGRA archives most of the activities happening at the sites through their logbooks. These may be complete from installation activities to noise hunting and mitigation works carried out during the lifespan of the observatory. Although there are site specific issues, often they encounter problems of similar nature and employing solutions that worked at the other sites may be a good strategy to start with. Also it is not uncommon to see previously fixed issues to reappear at a later time where the time scales could be of few months to years. This happens due to recurring environmental fluctuations and configuration changes in the detector. Since the current GW detectors aim at coincident detection of events, joint uptime of the instruments are crucial. This is more significant because the probability of detection scales linearly with observation time and cubically with sensitivity of the instrument.

The effective information extraction and processing of logbook information as envisaged here is expected to help in making better decisions pertaining to detector maintenance. For example, identifying the subsystems that could possibly get affected during instrument upgrade will be vital in scheduling and coordinating tasks among sub-groups involved. Similarly, long term tracking of a issue can be carried out to see if the various overhauling attempts indeed lead to an improvement in performance which correlates with lesser number of related posts.

In the case of GW interferometers, day-today activities are recorded using web interfaces known as Logbooks. It is mandatory for the reports to have a title, section, task, details and author details. Although anyone can view the reports, only users with valid credentials can login to add logs and additional supporting files like measurement figures, sensor data, codes etc. It is also possible to to add comments and carry out further discussion on any of the log book entries. Details of retrieved information are given below in Table 1

\section{HEY LIGO FUNCTIONALITIES}

An open access NLP based web application implementation named Hey LIGO is developed and deployed to support the commissioning and characterization efforts at the GW observatories. It relies on the logbook data recorded since 2010 by scientists specialized in different aspects of the detector. Every query is answered by matching it with most relevant logbook entries sorted as per their closeness to the query term in the word-vector space. We further analyze the sentiment of the post and color code so that green indicates a positive outcome and red corresponds to something undesirable in the context of activities carried out at the detector. An image retrieval facility displays thumbnail of the figures attached to the sorted data simplifying the knowledge discovery process. Contextual data visualization across multiple detectors is carried out as shown in Fig 6 and Fig 5. This feature lets the user to compare and see the trends in the searched keyword across different observatories.

Automatic check for new data entries is done periodically so that the NLP models are regularly updated. We track the volume of discussions happening on various topics and hence identify and rank the trending issues on a daily 
Table 1. Log book details retrieved from different GW observatories.

\begin{tabular}{|l|c|c|c|c|c|}
\hline Observatory & Logbook Entries & Contributors & Timespan & Dictionary Size & Clusters \\
\hline LIGO Livingston $^{\mathrm{a}}$ & 24351 & 261 & $2010-2017$ & 2273 & 455 \\
\hline LIGO Hanford $^{\mathrm{b}}$ & 24968 & 237 & $2010-2017$ & 2713 & 543 \\
\hline Virgo $^{\mathrm{c}}$ & 34592 & 660 & $2010-2017$ & 5026 & 1005 \\
\hline
\end{tabular}

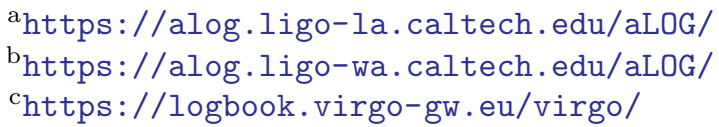

basis. Scientists involved with the project will mostly be interested in getting notified about specific issues that correlates with their domain of expertize and so the application only issues alerts to registered participants with matching interests. This targeted delivery will remove clutter and will ensure proper dissemination of information to the concerned people.

Code development is usually a tedious procedure wherein significant amount of time is spent on readability and re-usability so as to benefit a wider research community. Our application makes better use of this idea by auto-detecting and notifying the user about the presence of codes in the searched content. This feature we believe would simplify the procedure involved in result reproduction and its consequent independent verification.

To check the performance of our application, we analyzed six months of logbook data from LIGO Livingston and compared the NLP results and the actual relevant entries. Table 2 gives the performance of the our implementation for certain set of randomly chosen keywords. In most cases, the false alarms occur at the tail end of the search results which represent neighbors of least relevance in the $\mathrm{k}-\mathrm{NN}$ search. These can be removed either by setting a threshold on the similarity distance measure or by post-filtering the results by additionally comparing the content of each post. Currently, we have implemented the process of post-filtering to remove such post in the final web application. In the future we are planning to incorporate a mechanism that will make use of feedback received from the users and utilize it to improve the accuracy in retrieving relevant posts.

Table 2. Table showing some of the alog posts from May $1^{\text {st }}$ to June $30^{\text {th }}, 2017$.

\begin{tabular}{|l|l|l|l|l|}
\hline \multirow{2}{*}{ Keyword } & \multirow{2}{*}{$\begin{array}{l}\text { Log } \\
\text { book } \\
\text { Entries }\end{array}$} & \multicolumn{3}{|l|}{ Posts retrieved by NLP code } \\
\cline { 3 - 5 } & & Total & Relevant & Irrelevant \\
\hline Lock loss & 108 & 108 & 89 & 19 \\
\hline Earthquake & 83 & 94 & 80 & 14 \\
\hline $\begin{array}{l}\text { Charge } \\
\text { measurement }\end{array}$ & 62 & 65 & 58 & 7 \\
\hline Guardian & 55 & 65 & 55 & 10 \\
\hline Oplev & 63 & 61 & 48 & 13 \\
\hline $\begin{array}{l}\text { Calibration } \\
\text { lines }\end{array}$ & 55 & 52 & 45 & 7 \\
\hline
\end{tabular}

\section{INFERRING FROM LOGBOOK ENTRIES}

Once the relevant logbook entries are identified using the techniques mentioned above, their associated meta-data can be utilized to obtain several quantitative information about the topic of interest. 


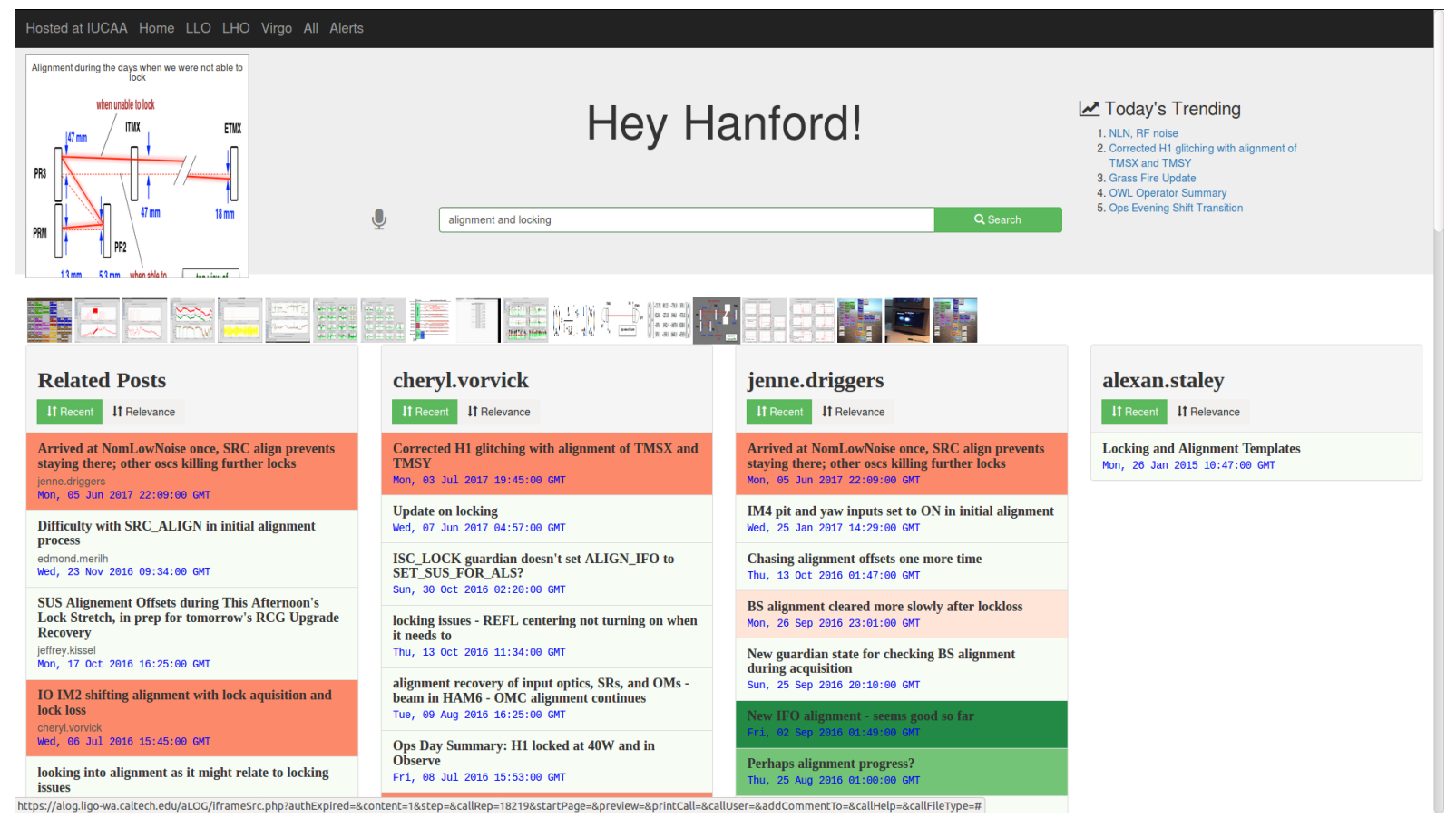

Figure 3. Screen-shot of Hey LIGO Web Interface. Search results are color tagged based on their overall sentiment. Trending posts are identified based on the associated meta-data consisting of comments \& discussions made within the LIGO community

\subsection{Trends within detectors}

Below we briefly compare the trends obtained for few test search queries and briefly discuss the observed patterns. Although of similar configuration, the effect of various noises on each detector seems to be of a different nature. Variation in instrumental behavior and environmental effects due to geographical location will also influence the efficiency of implemented mitigation measures. Figuring out such details can positively speed up the commissioning activities of future detectors like LIGO-India.

\section{Installation}

First plot from figure 6 shows the trends in posts related to installation work at each of the observatories. Activities picked up momentum in 2010 at LIGO and continued till the mid of 2014 after which testing and commissioning tasks started. Advanced Virgo seems to have started such activities in 2014 and carried on till the end of 2016.

\section{Jitter Noise}

Jitter noise arising out of laser pointing fluctuations (Martynov et al. 2016), is sensitive to cavity alignments and angular mirror motions. It has been partly caused by the pre-stabilized laser (PSL) periscope motion induced by chiller water flow around PSL's high power oscillator. Various efforts to understand it's possible origin and subsequent efforts to subtract it from the data stream is reflected through the increased number of alogs at the Hanford detector compared to other sites. Commissioners performed online feed-forward noise subtraction using auxiliary witness channels which reduced the coupling significantly (Sigg; Vajente; Driggers). 
3. Scattering Noise in LHO and LLO Noise from scattered light is currently one of the factors that limit the sensitivity in the frequency bin from $50 \mathrm{~Hz}$ to $200 \mathrm{~Hz}$ (see Fig. 4) especially during periods of high microseism. Off-axis beam scattered laser beam could hit a reflecting surface like camera mirror mount or beam tube and reenter the cavity. Nonlinear features are seen in the gravitational wave spectrum when this beam picks up resonances from reflecting surfaces which then get upconverted or phase modulated by lowfrequency seismic-like motion. It's effect at LLO is more pronounced as compared to LHO as the former is vulnerable to microseismic activity (Ottaway et al. 2012).

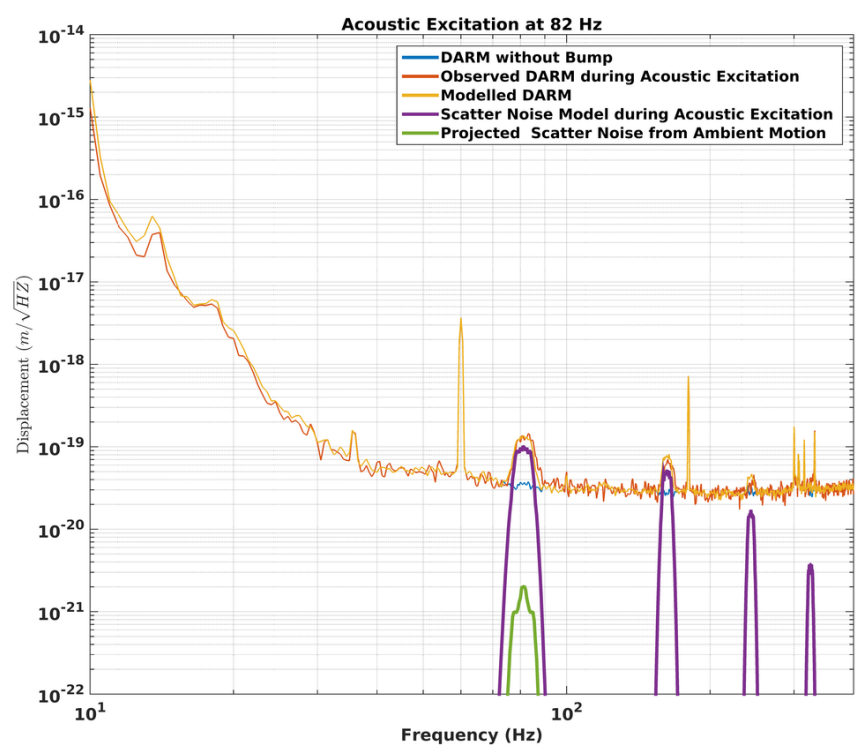

Figure 4. Noise due to scattered light observed at LIGO Livingston. Noise gets amplified and upconverted during periods of high microseism and limits the sensitivity range of the GW detectors.

Figure 4 shows the effect of acoustic excitation on $82 \mathrm{~Hz}$ peak seen in gravitational wave differential arm motion (DARM) data. The acoustic injections carried out at LIGO Y-end station are reconstructed using the proposed model (Accadia et al. 2010) Scatter Noise $=\mathrm{A}$ $\sin \left(4^{*}\right.$ pi* $^{*}\left(\mathrm{n}^{*}\right.$ Yrms + Yac $) /$ lambda $)$ where Yac $=\mathrm{B}^{*} \sin (2 \mathrm{pi}$ fo $\mathrm{t})$ where Yrms is the ground motion and Yac is the chamber motion with $(\mathrm{A}, \mathrm{n}, \mathrm{B})$ being the tunable parameters. Model parameters using are fine tuned using pattern search. The scatter noise projection to DARM from ambient motion is obtained by scaling down the chamber motion based on the accelerometer signal before and after injection.

\section{Non-astrophysical Transients}

Glitches often show up in the strain data leading to false alarms in the various search pipelines that look for astrophysical signals. Triggers are also observed in badly functioning instruments and are witnessed in auxiliary sensor channels. Some of them are also reported to cause loss of lock of the interferometer. The general operation of all the three detectors have been affected by such transients ever since their beginning of operation 6. The report generation feature of our application provides the following glitch distribution (Fig. 5) across multiple subsystems based on their tags in the data. It is interesting to note the subtle variations in the noise sources between LHO and LLO. The origin of many of them have been studied and reported in the logbooks while a vast majority are still not well understood.

\subsection{Visualizing Observatory as a Complex Network}

Behavior of an observatory and the elements that lead to changes within the system behavior can be studied through its representation as 

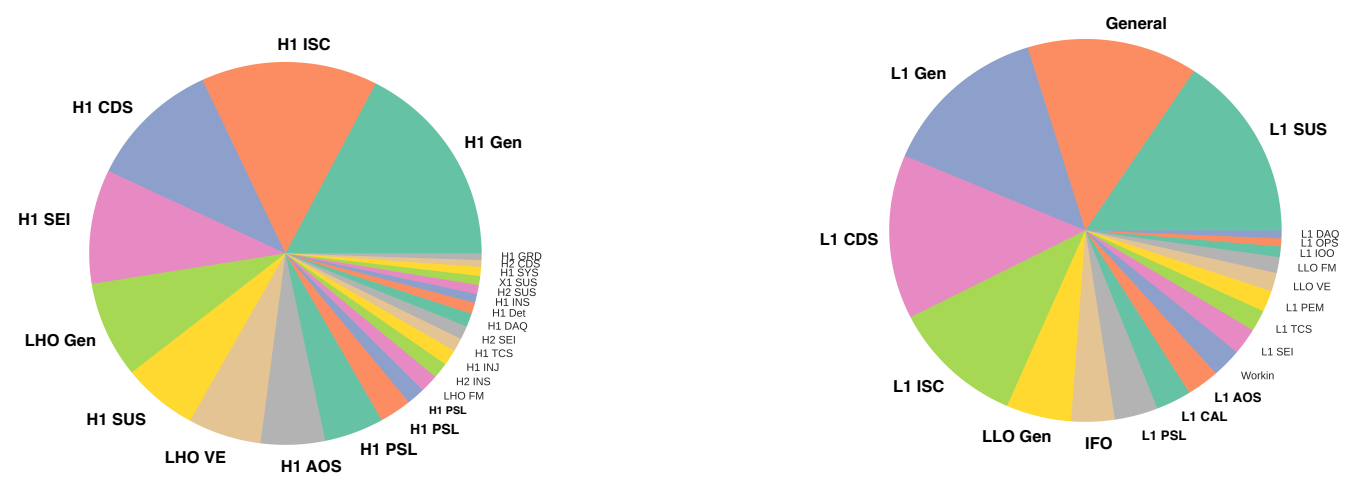

Figure 5. Pie Diagram shows how various LIGO subsystems contribute to the various non-astrophysical glitches seen in LIGO. Acronyms Used, ISC: Interferometer Sensing and Controls, CAL: Calibration, AOS: Auxiliary Optics Support, SUS: Suspension, VE: Vacuum Electronics, SEI: Seismic External Isolation, CDS: Control and Design System

a complex network. Complexity is expressed through nodes and links within the network. Here, the nodes can be either subsystems or specific instruments or even subgroups within the observatory and edges between them provide the probability of each one them being connected to the other as inferred from logbook entries. We first create a dictionary of subsystem keywords and for each one, find the frequency of their joint occurrence with everyone else. This information is then used to form the adjacency matrix whose diagonal elements are all zero and the off-diagonal value representing the linkage is given by the ratio of joint occurrence frequency divided by total occurrence of the keyword. Adjacency matrix being non-symmetric leads to a directed graph. Number of incident edges determines the node size while the edge width is given by the associated connection probability. To better aid visualization, we adopt Force Atlas 2 layout (Jacomy et al. 2014) with repulsion being approximated using Barnes Hut optimization (Barnes \& Hut 1986) which is well suited for larger graphs. The interconnectedness information within the observatory revealed through these networks may help in identifying the critical nodes in the system and the makes it easier to identify the vulnerable connections. These representations could possibly be useful during large scale repair and maintenance as they reveal the other subsystems that can get affected in the process.

In Fig 7 we show the network connection for a few prominent nodes of Virgo observatory. It differs from real-world networks in terms of its degree distribution (degree refers to the number of edges connected to each node). Sparse networks are characterized by a degree distribution which takes form of a power law and are commonly seen in biological networks and computer networks. (Barabási 2016). For the case of Virgo network, this distribution deviates from such a power law indicating dense connection between the nodes. Further research is needed to analyze the network and study the instrument's robustness to random sub-system failures.

\section{DISCUSSIONS \& CONCLUSION}

We have demonstrated how information retrieval and recommendation systems could be useful for LIGO like astronomical observatories. Compared with conventional search associated with the existing sites, our web applica- 

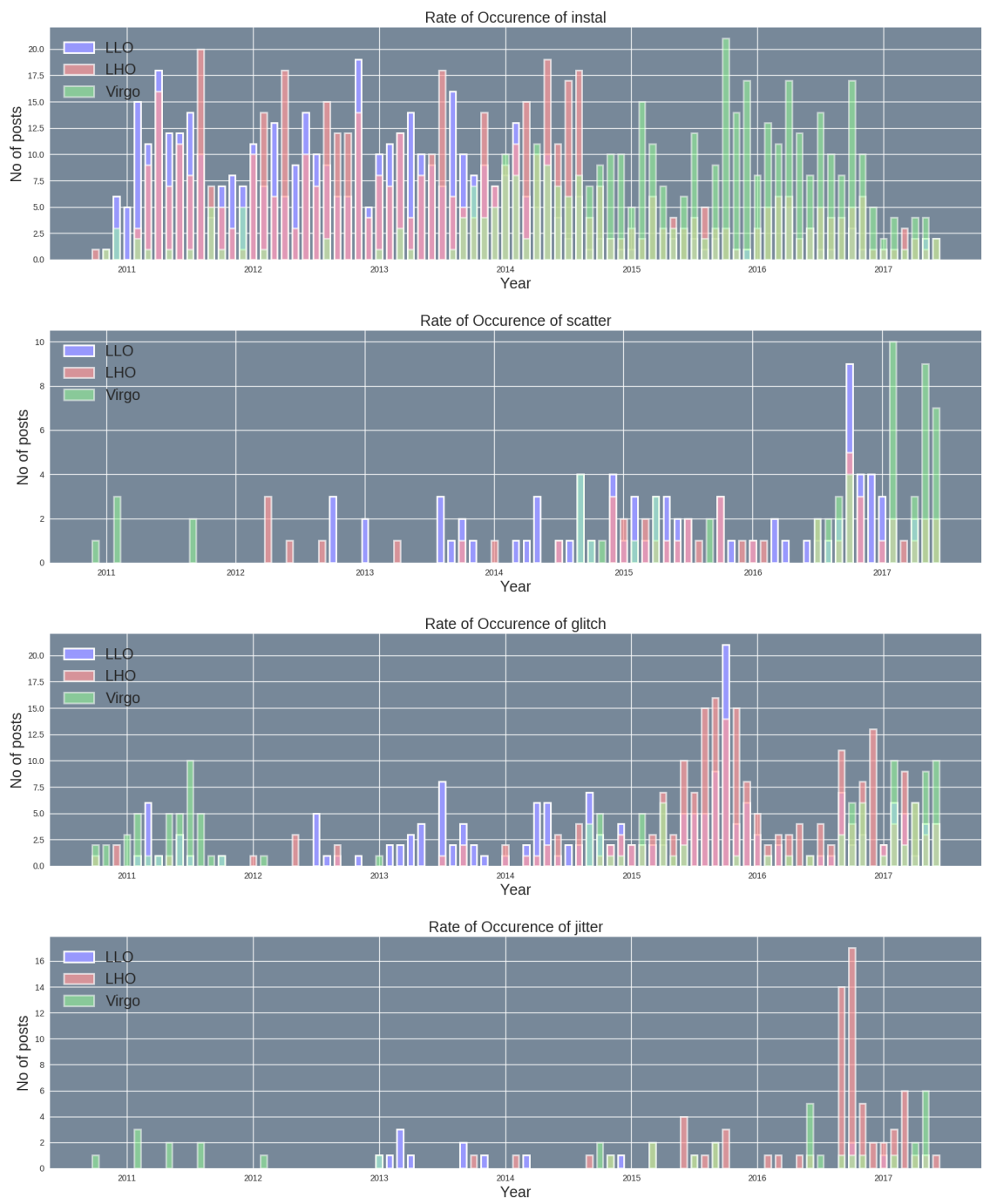

Figure 6. Rate of occurrence of different key words in multiple detectors are a function of time

tion incorporates a natural language processing based information retrieval system which can also do visualization of the user queried data. Involving a wider science community in big science projects can alleviate some of the issues related to lack of sufficient man power within the project. The developed interface identifies the major issues based on the discussions done within LIGO community and recognizes the trending issues. It is plausible that someone outside the project has already seen and solved these before. Hence proper dissemination of information will help in technical experts outside the project collaboration to contribute improving the overall performance of the instrument.

Coordinated efforts are being undertaken worldwide to carry out electromagnetic followup searches looking for counter parts to coalescing binaries sources(Abbott et al. 2016a). During the instance of GW candidate event alert, astronomers may be able to take advantage of our application and know more about the instrument.

Future improvement in the application would be to include capabilities wherein an identified issue will be provided with possible fixes making use past attempts which fixed an identical 

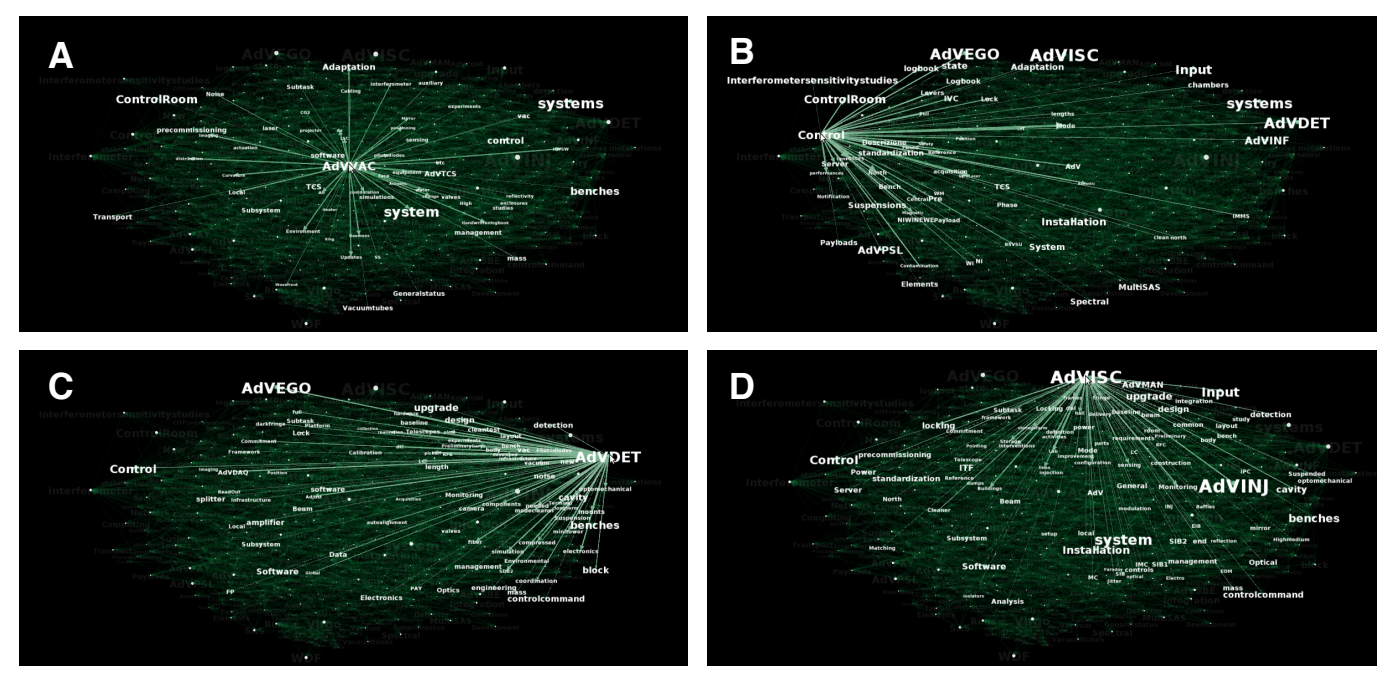

Figure 7. Network plot of the Virgo detector highlighting the inter-connections between various subsystems. Subplots highlight the directed graph for A) Vacuum, B) Control system, C) Photodetector and D) Interferometric sensing \& control.

issue. This would require text abstraction and summarization, quite challenging when the data has ample amount of technical terms. Efforts to add other GW detectors like GEO600 and KAGRA is currently under progress and will enhance the effectiveness of our application.

This kind of system has a lot of potential applications with the commissioning and running of large science projects like the SKA and future LIGO observatories. In this project the data source was more unstructured and had few tags related to the status of different activities. At present institutions like SKA South Africa which is in charge of building MeerKAT ${ }^{2}$ telescope which is one of the precursors to SKA, use a more structured systems like JIRA ${ }^{3}$ for issue tracking and $\log$ keeping ${ }^{4}$. Scaling our present system to such databases can improve the efficiency of topical modeling. This also enables auto-update of the learning database as more

\footnotetext{
2 http://www.ska.ac.za/science-engineering/ meerkat/

3 https://www.atlassian.com/software/jira

4 https://indico.skatelescope.org/event/402/ material/1/6.pdf
}

and more information is logged into the system finally making it a robust.

The aforementioned feature will eventually be very useful when the organization grows with the number of participants increasing over time. Availability of such systems will make the reusability of information much easier and efficient. This will also help in resolving instrument issues much easier and faster. Enhanced analytics of key components and recurring issues can help improving the fault tolerance of different subsystems and could provide insights on how modify them for better performance.

\section{ACKNOWLEDGEMENTS}

We would like to thank detector characterization group and machine learning sub-group of the LIGO Scientific Collaboration for their comments and suggestions. NM acknowledges the Council for Scientific and Industrial Research (CSIR), India for providing financial support as a Senior Research Fellow and Navajbai Ratan Tata Trust (NRTT) grant for supporting his visit to LIGO Livingston. A. K. Aniyan would like to thank the SKA South Africa postgraduate bursary program. SM acknowledges the support of the Science and Engineering Research 
Board (SERB), India through the fast track Grant No. SR/FTP/PS-030/2012. Authors express thanks to Arnaud Pele, Anamaria Effler and Ajit K Kembhavi for their valuable comments and suggestions. Authors wish to thank Malathi Deenadayalan and Santosh Jagade for technical support. LIGO was constructed by the California Institute of Technology and Massachusetts Institute of Technology with funding from the National Science Foundation and operates under Cooperative Agreement No.PHY0757058. This paper has been assigned LIGO Document No. LIGO-P1700250.

\section{REFERENCES}

Abbott, B., Abbott, R., Abbott, T., et al. 2016a, The Astrophysical journal letters, 826, L13

Abbott, B. P., et al. 2016b, Phys. Rev. Lett., 116, 061102. https://link.aps.org/doi/10. 1103/PhysRevLett.116.061102

Accadia, T., et al. 2010, Classical and Quantum Gravity, 27, 194011. http://stacks.iop.org/ 0264-9381/27/i=19/a=194011

Andoni, A., \& Indyk, P. 2006, in Proceedings of the 47th Annual IEEE Symposium on Foundations of Computer Science, FOCS '06 (Washington, DC, USA: IEEE Computer Society), 459-468. http://dx.doi.org/10.1109/FOCS.2006.49

Barabási, A.-L. 2016, Network science, Cambridge university press

Barnes, J., \& Hut, P. 1986, nature, 324, 446

Behrens, C. A., \& Bassu, D. 2006, Information retrieval and text mining using distributed latent semantic indexing, Google Patents, uS Patent 7,152,065

Bengio, Y., Ducharme, R., \& Vincent, P. 2003, Journal of Machine Learning Research, 3, 1137

Cointet, J.-P., \& Roth, C. 2010, in ICWSM

Driggers, J. 2017, LHO alog 34631, https://alog.ligo-wa.caltech.edu/aLOG/ index $\cdot$ php?callRep $=34631$

Fan, J., Han, F., \& Liu, H. 2014, National science review, 1, 293

Goldberg, Y., \& Levy, O. 2014, ArXiv e-prints, arXiv:1402.3722

Gretzel, U., Mitsche, N., Hwang, Y.-H., \& Fesenmaier, D. R. 2004, Information Technology \& Tourism, 7, 3

Han, J., Pei, J., \& Kamber, M. 2011, Data mining: concepts and techniques (Elsevier)

Harris, D., \& Harris, S. 2012, Digital design and computer architecture (Elsevier)
Hassan, A., Radev, D., Cho, J., \& Joshi, A. 2009, Ann Arbor, 1001, 48109

Hu, H., Wen, Y., Chua, T.-S., \& Li, X. 2014, IEEE Access, 2, 652

Huang, C.-R., Šimon, P., Hsieh, S.-K., \& Prévot, L. 2007, in Proceedings of the 45th annual meeting of the ACL on interactive poster and demonstration sessions, Association for Computational Linguistics, 69-72

Jacomy, M., Venturini, T., Heymann, S., \& Bastian, M. 2014, PloS one, 9, e98679

Kerzendorf, W. E. 2017, ArXiv e-prints, arXiv:1705.05840

Khan, N., Yaqoob, I., Hashem, I. A. T., et al. 2014, The Scientific World Journal, 2014

LaValle, S., Lesser, E., Shockley, R., Hopkins, M. S., \& Kruschwitz, N. 2011, MIT sloan management review, 52, 21

Leskovec, J., Rajaraman, A., \& Ullman, J. D. 2014, Mining of massive datasets (Cambridge University Press)

Li, Y., Xu, L., Tian, F., et al. 2015, in IJCAI, 3650-3656

Liang, T.-P., Lai, H.-J., \& Ku, Y.-C. 2006, Journal of Management Information Systems, 23, 45

Ling, W., Dyer, C., Black, A. W., \& Trancoso, I. 2015, in HLT-NAACL, 1299-1304

Markov, Z., \& Larose, D. T. 2007, Data mining the Web: uncovering patterns in Web content, structure, and usage (John Wiley \& Sons)

Martynov, D. V., et al. 2016, Phys. Rev. D, 93, 112004. https://link.aps.org/doi/10. 1103/PhysRevD.93.112004

Mikolov, T., Chen, K., Corrado, G., \& Dean, J. 2013a, ArXiv e-prints, arXiv:1301.3781

Mikolov, T., Sutskever, I., Chen, K., Corrado, G., \& Dean, J. 2013b, ArXiv e-prints, arXiv:1310.4546 
Miner, G., Elder IV, J., \& Hill, T. 2012, Practical text mining and statistical analysis for non-structured text data applications (Academic Press)

Morin, F., \& Bengio, Y. 2005, in Aistats, Vol. 5, 246-252

Nielsen, F. A. 2011, AFINN, Richard Petersens Plads, Building 321, DK-2800 Kgs. Lyngby: Informatics and Mathematical Modelling, Technical University of Denmark. http://www2.imm.dtu.dk/pubdb/p.php?6010

Ottaway, D. J., Fritschel, P., \& Waldman, S. J. 2012, Opt. Express, 20, 8329.

http://www .opticsexpress.org/abstract. cfm?URI=oe-20-8-8329

Pazzani, M., \& Billsus, D. 2007, The adaptive web, 325

Ricci, F., Rokach, L., \& Shapira, B. 2011, Introduction to recommender systems handbook (Springer)
Sigg, D. 2016, LHO alog 30412, https://alog.ligo-wa.caltech.edu/aLOG/ index $\cdot$ php?callRep $=30412$

Sigurbjörnsson, B., \& Van Zwol, R. 2008, in Proceedings of the 17th international conference on World Wide Web, ACM, 327-336

Smirnov, I. 2008, Mechanical Translation, 52

Stephens, Z. D., Lee, S. Y., Faghri, F., et al. 2015, PLoS Biol, 13, e1002195

Vajente, G. 2016, LHO alog 30473, https://alog.ligo-wa.caltech.edu/aLOG/ index $\cdot$ php? callRep $=30473$

Wu, X., Zhu, X., Wu, G.-Q., \& Ding, W. 2014, ieee transactions on knowledge and data engineering, 26, 97

Zoghbi, S., Vulić, I., \& Moens, M.-F. 2013, in Proceedings of the 2013 international workshop on Mining unstructured big data using natural language processing, ACM, 45-52 